\title{
Fare o disfare il genere: la tentazione paradossale della collana di racconti lesbici Principesse azzurre
}

Faire ou défaire le genre : la tentation paradoxale de la collection de récits lesbiens Principesse azzurre

\section{Alison Carton-Vincent}

\section{(2) OpenEdition}

\section{Journals}

Edizione digitale

URL: http://journals.openedition.org/cei/1269

DOI: $10.4000 /$ cei. 1269

ISSN: 2260-779X

\section{Editore}

UGA Éditions/Université Grenoble Alpes

Edizione cartacea

Data di pubblicazione: 30 juin 2013

Paginazione: 253-269

ISBN: 978-2-84310-245-5

ISSN: 1770-9571

\section{Notizia bibliografica digitale}

Alison Carton-Vincent, «Fare o disfare il genere: la tentazione paradossale della collana di raccont lesbici Principesse azzurre», Cahiers d'études italiennes [Online], 16 | 2013, online dal 15 décembre 2014, consultato il 26 mars 2021. URL: http://journals.openedition.org/cei/1269 ; DOI: https://doi.org/ 10.4000/cei. 1269 
FARE O DISFARE IL GENERE:

LA TENTAZIONE PARADOSSALE DELLA COLLANA

DI RACCONTI LESBICI PRINCIPESSE AZZURRE

\author{
Alison Carton-Vincent \\ Université de Provence
}

La collana delle Principesse azzurre è una serie di raccolte di racconti ${ }^{\mathrm{I}}$ lesbici attualmente composta di sette volumi. La prima raccolta, intitolata Principesse azzurre. Racconti d'amore e di vita di donne tra donne è pubblicata nel 2003 da Mondadori. Da allora, ogni anno esce un volume, sempre curato da Delia Vaccarello, scrittrice, giornalista e attivista, con un titolo che contiene sempre il sintagma "principesse azzurre», tranne l'ultimo volume del 2009 (Pressoché amanti. Racconti d'amore e di vita di donne tra donne). Ogni raccolta è costituita di un numero di testi variabile (da undici a ventitré), scritti da autrici molto diverse, tanto per il loro percorso individuale quanto per la loro pratica di scrittura, sicché testi di autrici affermate si alternano a quelli di illustri sconosciute (che usano il loro nome o uno pseudonimo).

La struttura di una raccolta si organizza sempre allo stesso modo. Prendiamo l'esempio della prima raccolta che contiene le seguenti rubriche: copertina e illustrazione, pagina di presentazione (titolo, sottotitolo, curatrice), introduzione di Delia Vaccarello (con un titolo che mira a dare un senso generale alla raccolta, come Coralità, Visibilità, Pressapoco...), ringraziamenti, titolo di «sezione» ${ }^{2}$, testi, nuova sezione, testi ecc., biografia

I. Mentre i testi sono per la maggior parte dei racconti, in realtà ci sono anche alcuni fumetti e brevi opere teatrali. Ma si tratta pur sempre di forme brevi, di qualche pagina.

2. L'organizzazione tematica interna non sarà più utilizzata nei volumi ulteriori, probabilmente perché quelli saranno dedicati ognuno a un tema particolare. Tale cambiamento corrisponde tra l'altro all'evoluzione della politica editoriale e del contesto socio-culturale: mentre i primi volumi mirano a colmare un vuoto culturale raccogliendo voci sparse e di solito non pubblicate, il successo e la visibilità crescenti della collana permettono una più grande selettività delle ultime raccolte e la scelta di un tema federatore. 
delle scrittrici, indicazioni bibliografiche delle opere lesbiche pubblicate in Italia ${ }^{3}$, indice. Lo scopo della pubblicazione si legge nella struttura delle raccolte: dare visibilità alla letteratura lesbica, attraverso un atto creativo con i racconti e memoriale con le bibliografie selettive; presentare le autrici lesbiche o che scrivono del lesbismo (poiché l'orientamento sessuale delle scrittrici non è un criterio di selezione) ${ }^{4}$ con l'aggiunta di biografie spesso divertenti; dare accesso a un universo culturale lesbico variegato con la giustapposizione tematica dei racconti e la messa in rilievo delle loro caratteristiche comuni nelle introduzioni.

Sette raccolte, centoventotto testi, sessantanove autrici, sette anni: Principesse azzurre è un magma narrativo proteiforme di cui non si può fare un'analisi sistematica in questo articolo. Tuttavia in questa sede cercheremo di leggere la serie delle Principesse azzurre nell'ottica di genere e delle sue rappresentazioni: poiché i testi mettono in scena delle donne, per lo più lesbiche, la questione di genere e dell'orientamento sessuale è onnipresente, dall'allusione rapida alla riflessione teorica. Siccome non rispettano l'equazione sesso femminile $=$ genere femminile $=$ sessualità eterosessuale e desiderio per gli uomini, le protagoniste di Principesse azzurre infrangono socialmente le leggi di genere. Questa trasgressione e questo scarto dalla norma si esprimono nella materia-testo. Vedremo allora come si intrecciano genere, lingua e letteratura. In quanto soggetti 'accanto' alla norma (o «eccentrici» per dirla con Teresa de Lauretis) ${ }^{5}$ ma che vivono in una società retta dalle regole di genere, le protagoniste (e narratrici) sembrano in preda a un dissidio tra desiderio di liberazione e di decostruzione delle norme di genere, e una sottomissione a volte inconscia ai canoni della mascolinità e della femminilità. Percorrendo le diverse tappe di formazione identitaria rappresentate nei racconti (l'infanzia, il rapporto con la madre, la scelta della maternità), proporremo un'analisi del rapporto alle norme delle nostre "principesse azzurre», discriminate due volte in quanto donne e in quanto omosessuali. Poi, ci soffermeremo sulle modalità di rappresentazione di sé delle protagoniste-narratrici nella collana, che usa la ripresa e la deco-

3. Anche la parte d'informazione bibliografica viene soppressa negli ultimi volumi. Desiderio di non ripetersi ogni anno o segno di un accesso ormai più facile a questi testi, per cui non è più necessario farne un elenco? Lo sviluppo di internet rappresenta un fattore d'importanza: la curatrice stessa dà il suo indirizzo e-mail nelle raccolte, l'indirizzo del suo sito, ma anche indirizzi di siti specializzati, in particolare nel secondo volume con una parte intitolata Link di carta.

4. Delia Vaccarello, Introduzione: Miracoli, in Principesse azzurre 2. Racconti d'amore e di vita di donne tra donne, a cura di Delia Vaccarello, Milano, Mondadori, 2004, p. 7: «Non mi interessava e non mi interessa che a scrivere sul tema - l'amore e la vita tra donne — fossero le donne lesbiche e basta. Mi preme dare fiato a questa dimensione scovandone la cornice culturale che prescinde dall'orientamento sessuale di chi ne scrive.»

5. Teresa de Lauretis, Soggetti eccentrici, Milano, Feltrinelli, I999. 
struzione di miti culturali e letterari, come quello dell'androgino. Infine, concluderemo sul rapporto tra letteratura e politica in questi testi che costituiscono una letteratura dissenziente dal punto di vista del genere ma che sono pubblicati comunque da una delle più famose case editrici italiane, Mondadori.

\section{Genere, lingua e letteratura}

«Credo che non si possa esistere senza farsi accogliere dalle storie. Che siano fiabe, racconti, romanzi, fumetti o canzoni, che siano vere o verosimili, le storie siamo noi. Senza, non siamo.» ${ }^{6}$ Queste le parole scelte da Delia Vaccarello per introdurre la primissima raccolta. Ricollegano vita e storia, esistenza e letteratura, in termini di assoluta necessità: senza letteratura, niente esistenza, senza rappresentazione di sé, niente sé. Ciò che può sembrare un'asserzione aleatoria in termini assoluti assume però un senso quando la si applica al caso delle minoranze, per giunta delle minoranze poco visibili: senza rappresentazione lesbica in una società eteronormativa, come essere lesbica, come dichiararsi lesbica? Identità e letteratura assumono una valenza particolare nel caso della letteratura omosessuale ma si pone allora la questione del modo di espressione di questa identità 'altra'. Come esprimersi nella sua differenza pur usando una lingua e una letteratura esogene? In quale rapporto con la tradizione linguistica e letteraria si collocano le "principesse azzurre»?

\section{Modalità narrative}

La narrazione non presenta segni distintivi particolari nella collana, tranne una grandissima variabilità. Si alternano narrazioni in terza e in prima persona, come si alternano narratrici omo- ed eterodiegetiche ${ }^{7}$. Ma va appunto notata questa assenza di caratterizzazione, se si paragona questa produzione con la produzione femminile del secondo Novecento e dell'inizio del xxi secolo. Se la definizione e l'affermazione dell'esistenza di una «scrittura femminile» rimangono problematiche, ciò non toglie che gli scritti

6. Delia Vaccarello, Introduzione: La coralità dell'intramare, in Principesse azzurre. Racconti d'amore e di vita di donne tra donne, Milano, Mondadori, 2003, p. 5.

7. Altre forme enunciative esistono nella raccolta, come nel racconto L'insostenibile pesantezza dei vent'anni, scritto alla seconda persona del singolare, con una voce narrante eterodiegetica (ibid., pp. 199-2I9). 
femminili hanno spesso in comune un'enunciazione in prima persona ${ }^{8}$. Invece qui non è il caso. Si tratterà di una caratteristica inerente alla forma del 'racconto'? O si deve ritenere la letteratura lesbica non un 'sottogruppo' della letteratura femminile bensì un gruppo a sé stante, in una logica di tipo wittighiano secondo la quale «les lesbiennes ne sont pas des femmes»"? Mentre la mole di scritti femminili in prima persona pubblicata sin dagli anni Sessanta-Settanta ha accompagnato e rafforzato la costruzione di un'identità femminile nuova, ci possiamo chiedere se l'importante presenza di narrazioni in terza persona o di racconti in cui la narrazione è eterodiegetica non fosse il segno di un'identità lesbica ancora in costruzione, non in quanto identità unica, ma in quanto identità accettata e visibile. Questa possibilità sembra confermata dall'evoluzione della dominante enunciativa nel corso degli anni: mentre diventano sempre più attivi i movimenti omosessuali italiani tra il 2003 e il 2009 (si pensi alla campagna per i PACS poi DiCo, ai Gaypride...), la narrazione si fa sempre più personale, per sboccare in un'ultima raccolta interamente in prima persona, in cui la nozione di testimonianza è centrale: "Questa è la novità della nuova raccolta Principesse azzurre che leggete. Racconti sì, pieni di vita. Testimonianze in prima persona che affiancano narrazioni di autrici esperte.» ${ }^{\mathrm{IO}}$ La tendenza ad un «io» "che non osa dire il proprio nome» per dirla con Oscar Wilde, a un «chi non dice il suo nome dicendo l'amore», per dirla stavolta con Eleonora Pinzuti in un suo articolo dedicato alla letteratura lesbica italiana ${ }^{\text {II }}$, sembra assottigliarsi (come il numero degli pseudonimi a favore dei nomi anagrafici delle autrici — o di firme verosimili di tipo nome-cognome) ${ }^{\mathrm{I2}}$.

8. Si veda Sabina Gola, La forme autobiographique dans la littérature féminine depuis les années soixantedix, Actes de la conférence internationale «La valeur de la littérature pendant et après les années 1970 : le cas de l'Italie et du Portugal", II-I3 mars 2004, Utrecht, Pays-Bas. Sul sito: <http: //congress7o.library.uu.nl/index. html?oooor2/index.html> (data di ultima consultazione Io febbraio 2013).

9. Monique Wittig, La pensée straight, Paris, Éditions Amsterdam, 2007, p. 6I.

Io. Delia Vaccarello, Introduzione: Sortilegio, in Pressoché amanti. Racconti d'amore e di vita di donne tra donne, a cura di Delia Vaccarello, Milano, Mondadori, 2009, p. 8.

II. Eleonora Pinzuti, La narrativa lesbica nel contesto europeo. Note al margine, in "Cahiers d'études italiennes. Novecento... e dintorni", 7, 2008, p. 213.

I2. Nella prima raccolta, sulle quindici autrici citate, quattro usano apertamente uno pseudonimo, una usa un semi-pseudonimo (cambia il suo nome per diventare "Marc de' Pasquali»), mentre nell'ultima, sulle tredici autrici, c'è un unico chiaro pseudonimo ("A.S. Laddor", di cui si legge nella bibliografia "Laddor è un acronimo. "La donna dagli occhi rossi"” (Pressoché amanti. Racconti d'amore e di vita di donne tra donne, cit., p. 2I7). 


\section{Lavorare la materia-lingua: creazioni linguistiche e incongruità di una definizione di sé in termini binari ed esclusivi}

Il legame tra identità ed espressione testuale di sé diventa più complesso quando il soggetto narrante o narrato non riesce a collocarsi nella solita binarietà dei generi uomo-donna. Le narratrici delle Principesse azzurre provano diversi procedimenti linguistici per denunciare l'incompatibilità del sistema grammaticale (femminile $o$ maschile) con la complessità dell'identità sessuale (concepita come continuum dal femminile al maschile, come presenza simultanea del maschile $e$ del femminile).

Nel racconto Annegamilamente, l'indeterminatezza di genere viene trascritta da una doppia marca grammaticale: la lettera simbolica "X» e il pronome relativo invariabile «chi», ma anche dalla doppia possibilità di accordo al maschile o al femminile:

«Sei scesa/o nelle ruvide pareti del mio sentire.» Questo il messaggio per «X». Ma non sa come navigarlo negli affollati canali eterei di Internet, né a chi telefonare, né a chi scrivere. Le lente ventose della sua mente non trovano ove assorbire soluzioni. Non le resta che sperare in un prossimo contatto "aperto»: percepisce in un breve senso di vuoto che "Xx» è padrone/a della situazione e che vuole sofferenza. ${ }^{13}$

Questo scarto tra espressione della complessità del genere e binarietà del linguaggio diventa anche fonte di grande umorismo, prova della sua assurdità, come nella commedia breve L'estate scorsa giù al ghetto ${ }^{\mathrm{I4}} \mathrm{di}$ Margherita Giacobino. Lorena, «accademica in carriera», esclama guardando Ivana «esemplare di butch italica»: "Non conosco nessun Giosuè. Comunque quella lì, quella scimpanzé, quella invece la conosco, me la ricordo benissimo, stava al convegno GLBTQNSCV di Bologna, e durante il mio intervento su Critica decostruttiva come autoerotismo applicato al testo non ha fatto altro che disturbare. ${ }^{15}$ Grazie al suo personaggio, l'autrice prende chiaramente in giro la sigla in perpetua espansione che viene usata per indicare la comunità dei gay, lesbiche, bisessuali, trans, queer o "questioning» (quelli/e che interrogano la loro identità sessuale), ecc., che intende riassumere la diversità degli orientamenti sessuali e delle identità sessuate con un'unica parola.

13. A. S. Laddor, Annegamilamente, in Principesse azzurre. Racconti d'amore e di vita di donne tra donne, cit., p. 124 .

I4. Margherita Giacobino, L'estate scorsa giù al ghetto, in Principesse azzurre 3. Racconti d'amore e di vita di donne tra donne, a cura di Delia Vaccarello, Milano, Mondadori, 2005, pp. $24 \mathrm{I}-272$.

I5. Ibid., p. 252. 


\section{Genere-gender egeneri letterari}

Procedimento frequente nella raccolta, il rimaneggiamento dei generi letterari permette alle autrici di confrontarsi con maggiore efficacia alle tensioni tra genere-gender e rappresentazione letteraria. La forma 'racconto' si dirama in due riscritture generiche principali: il racconto di anticipazione e la fiaba, tutti e due segnati dalla loro ambientazione non-realistica.

Il racconto Annegamilamente è un caso interessante di espressione del genere-gender tramite la letteratura d'anticipazione, più esattamente la distopia ${ }^{16}$. La protagonista, Spira, vive in una società retta dalla "Regola», stabilita dai «Pensanti». Grazie alle nuove tecnologie, può cambiare apparenza, voce, odore e "musica d'accompagnamento». Questo gioco con il corpo e l'aspetto è una costante del racconto: Spira sperimenta diverse apparenze, e la narratrice presenta le leggi che regolano questi cambiamenti e i rischi incorsi da chi li trasgredisce. L'uso del genere «racconto di anticipazione» sottolinea quanto le apparenze, e per estensione dei simboli di genere, siano molteplici e non definiscano una identità immutabile bensì molteplice. La scelta di questo genere letterario permette la messa in scena di un essere mutevole, lungi dalle rappresentazioni idealizzate di donne di una data letteratura mascolina precedente. Già presente nella letteratura femminista del secondo Novecento, questa tendenza a impadronirsi della propria rappresentazione in letteratura e a esprimere un'identità complessa giunge qui al proprio culmine tramite l'uso della fantascienza, nella misura in cui essa potenzia la capacità sovversiva della letteratura: se l'arte ha la capacità di mostrare un altro possibile con la finzione, il ventaglio di questi possibili aumenta considerevolmente quando sparisce il vincolo del verosimile.

La fiaba è il secondo genere letterario rivisitato dalla collana. Scegliendo di parodiare un genere tanto codificato ${ }^{17}$, Principesse azzurre diventa subito sovversivo rispetto alle norme, quelle di genere in particolare, poiché si tratta di scardinare il fondamento stesso della fiaba: la presenza di un principe e di una principessa (o assimilato/a: re, regina) eterosessuali, a favore di una coppia (potenzialmente) omosessuale di principesse. «Svegliare la bella addormentata è un atto di ribellione? Se a farlo è il bacio di una prin-

I6. La volontà di reggere il mondo in vista del bene comune dei «Pensanti» porta ad aberrazioni dal punto di visa umano, per esempio per quanto riguarda l'amore: "Chissà se erano state capaci di contenere il sentimento e rispettare le 48 ore di tempo che i Pensanti concedono alle coppie per vivere la passione privata fuori dai locali adibiti all'amore. Sì, per regola spingersi oltre diventava pericoloso e fonte di legami troppo forti: la disciplina di controllo emotivo, per quanto produttiva, aveva bisogno di essere aiutata da situazioni di basso stress.» (A.S. Laddor, Annegamilamente, cit., p. I2I.)

17. Si veda per esempio Vladimir Propp, Morfologia della fiaba, Torino, Einaudi, 1966 (edizione originale 1928). 
cipessa azzurra potrebbe trattarsi di una variante eversiva della fiaba», ricorda Delia Vaccarello nella terza raccolta della serie ${ }^{18}$. Proprio in questo volume, il tema della fiaba dissenziente si diffonde attraverso tre elementi principali: il titolo, la copertina, e il racconto finale $A z z u r r a$. Il titolo, Principesse azzurre, colloca di primo acchito la raccolta in una posizione ironica rispetto alla tradizione culturale e funziona "come segnale quasi provenzale di presa di distanza» ${ }^{19}$. L'illustrazione presenta due giovani principesse, tipo Walt Disney, descritta da una delle autrici del volume, Rosanna Fiocchetto, come una "copertina, ironica e fiabesca, in cui una donna vestita d'azzurro in groppa ad un cavallo dall'occhio tra furbo e meravigliato invita al viaggio amoroso, sullo sfondo di un castello lontano, un'altra donna in abito rosa e viola $»^{20}$. Le due principesse sono bionde $\mathrm{e}$ vestono bellissimi vestiti, insomma possiedono tutti gli attributi tradizionali del loro genere. Eppure si danno la mano. L'intento è chiaro: si tratta di dare vita all'immaginario lesbico, tramite rappresentazioni (visuali o testuali) in cui riconoscersi. La raccolta si conclude con un racconto il cui titolo, Azzurra, rinvia all'universo del meraviglioso (dal "principe azzurro» alla «fata dai capelli turchini» di Collodi). Il valore simbolico della fiaba e lo scarto ironico vengono sottolineati dall'onomastica, poiché la principessa si chiama "Azzurra»" ${ }^{21}$. Dopo un incipit, la bella principessa è a confronto con il proprio destino: quando si guarda allo specchio, non vede la sua immagine riflessa. L'unica soluzione è l'amore ("Quando Azzurra s'innamorerà, vedrà nella persona amata la sua immagine» $\left.{ }^{22}\right)$. Per il tramite di un animale favoloso (un pesce-luna parlante), Azzurra incontra l'amore nella persona di una principessa addormentata, che si sveglia al suo contatto, e «da allora Azzurra e Nerina vissero felici» ${ }^{23}$. L'autrice, Vanessa Ambrosecchio, propone una fiaba lesbica ma con un lieto fine, segno che la trasgressione delle norme può non essere fatale, anzi può essere fonte di felicità per una principessa, e ovviamente per la bambina che non mancherà di identificarsi con essa.

18. Delia Vaccarello, Introduzione: Principesse messe a nudo, in Principesse azzurre 3. Racconti d'amore e di vita di donne tra donne, cit., p. 5 .

19. Eleonora Pinzuti, La narrativa lesbica nel contesto europeo, cit., p. 2 I2.

20. Rosanna Fiocchetto, La terza volta delle Principesse, articolo online sul sito della Libreria delle donne di Milano: <www.libreriadelledonne.it/Stanze/Paradiso/Nespole/fiocchetto.htm> (data di ultima consultazione Io febbraio 2013).

2I. Questo procedimento è usato allo stesso modo in un'altra parodia della fiaba tradizionale, il film d'animazione Shrek 2, in cui il principe azzurro si chiama «Azzurro».

22. Vanessa Ambrosecchio, Azzurra, in Principesse azzurre 3. Racconti d'amore e di vita di donne tra donne, cit., p. 306.

23. Ibid., p. 3 IO. 


\section{«Donna non si nasce: lo si diventa.» La questione del rapporto alle norme}

Principesse azzurre si interroga su come si relazionano le donne omosessuali alla norma: norma letteraria ma anche sociale. Tre tappe particolari della formazione identitaria femminile sono ricorrenti: l'infanzia, il confronto con il modello materno e la scelta della maternità.

\section{«Dalla parte delle bambine»: genere e infanzia}

Già analizzato nel 1973 da Elena Gianini Belotti nel saggio Dalla parte delle bambine, il carattere precoce dell'apprendimento delle ingiunzioni di genere viene illustrato dai racconti di Principesse azzurre che mettono in scena protagoniste molto giovani a confronto con norme tanto incomprensibili quanto impossibili da trasgredire. In Vita all'aria aperta, due gemelli, una bambina (Violetta) e un bambino (Fiore), scoprono sin dalla loro nascita che, nonostante la loro somiglianza totale, la gente si aspetta da loro che siano diversi poiché non sono dello stesso sesso. Eccone l'incipit:

Gemelli. Uguali come due gocce d'acqua. Come due violette, due fiorellini, disse il padre, e questo ne destinò il nome.

Come due violette, certo, disse la madre, però una differenza c'è, e che cosa fa più differenza di questa? E indicò al marito, inebetito davanti a quei due corpicini nudi, la patatina dell'uno e il pisellino dell'altro. ${ }^{24}$

Ma la differenza degli organi genitali non è sufficiente e rivela invece la sua poca significanza: i bambini si burlano della madre scambiando le loro identità, e la madre prova con accanimento a rendere effettivo il lavoro di «sessaggio» dei piccoli individui:

Il problema di riconoscerli a colpo d'occhio era stato risolto con il colore delle tutine (rosa lei, azzurro lui), scherzi a parte del primogenito che più di una volta riuscì a far confondere la madre. [...] Fece tagliare cortissimi i riccioli di Fiore, lasciò lunghi quelli della bambina con l'aggiunta di un fiocchetto rosa. ${ }^{25}$

Ciononostante, i bambini non capiscono i motivi di questi trattamenti opposti. Usando un narratore eterodiegetico che si accontenta il più delle volte di riportare i fatti senza commentarli, le norme di genere sembrano ancora più assurde e inique. Così, quando la maestra vuole separare gli

24. Sara Zanghì, Vita all'aria aperta, in Principesse azzurre 2. Racconti d'amore e di vita di donne tra donne, cit., p. 6I.

25. Ibid., p. 62. 
alunni tra maschi e femmine, il narratore descrive il loro stupore in modo poetico:

No, no, non è il tuo posto. Tu devi sedere nel lato dei maschietti, là.

I bambini la guardarono e la maestra vide in quei grandi occhi innocenti d'un azzurro limpido come il cielo in certe mattine d'aprile una muta e dolorosa meraviglia. ${ }^{26}$

Il modello della fiaba (narrazione eterodiegetica, somiglianza incredibile, poeticità e poca storicizzazione del racconto) permette di nuovo di scrivere del genere in letteratura senza inclinare al saggio teorico ma sottolineando comunque la violenza delle norme di genere e la loro arbitrarietà.

\section{Il modello materno}

I rapporti madre-figlia sono una tematica ricorrente della letteratura femminile, in modo particolare nella produzione femminista: diventare donna in un modo diverso (scegliendo di non sposarsi, di non avere bambini o in un'unione libera e paritaria per esempio) rende necessaria una presa di distanze e una critica del modello rappresentato dalla propria madre. Lo stesso accade in Principesse azzurre, in cui le protagoniste, per via del loro orientamento sessuale e a volte per via del non-rispetto delle convenzioni del genere femminile, intrattengono rapporti complessi con la madre. La questione della norma materna offre lo spunto a testi a turno giocosi, inquietanti o nostalgici. In Le parole che «lei» mi ha detto, la narratrice si rivolge alla madre e ripercorre con tono umoristico le loro divergenze di opinioni sull'omosessualità:

Cara mamma, volge il terzo anno da quando decisi di lasciare tutto per diventare lesbica. Ricordi? Ne parlavo sempre da piccola [...].

Lo so, eri preoccupata, ricordo le interminabili discussioni a tavola: «Le lesbiche non hanno futuro, non è roba per ragazze di buona famiglia. Che dirà la gente? Tua cugina ha aperto un negozio di erboristeria e a maggio si sposa. Tesoro della mamma, ti apro un negozio di vestiti usati indiani, un ristorante di cibi macrobiotici; quello che vuoi, ma lesbica no. Non è un mestiere sicuro, devi stare sempre lontana da casa, vivere tra gente sconosciuta, andare in giro la notte, frequentare locali equivoci..."

Mammina cara, lo sai che sono ostinata: lesbica volevo essere e lesbica sono diventata! ${ }^{27}$

Invece il tono si incupisce in Prigioniere, racconto in cui la narratrice si rivolge anche lei alla madre, mentre guarda una foto della madre da giovane. Torna su un aneddoto doloroso che chi legge capisce a poco a poco: la narratrice si sarebbe sottoposta ad analisi genetiche per 'capire'

26. Ibid., p. 63 .

27. Iceblues, Le parole che «lei» mi ha detto, in Principesse azzurre 2, cit., pp. 97-98. 
l'origine della propria omosessualità. Commenta con tristezza la reazione della madre:

«In effetti sei un essere umano di quarantasei cromosomi, non un primate, pensa, dai tuoi modi da animale selvaggio, non l'avrei mai detto.» Sorridevi perché leggendo nel referto la conferma che tutto fosse a posto, ti levavi il peso di non avermi fatto sana, giusta, normale. Sarebbe bastato un salto di vent'anni e ciò che sono non ti avrebbe ferita più. Ma allora, allora eri prigioniera quanto me. Se una figlia si sente prigioniera anche la madre lo è. ${ }^{28}$

Che la tonalità sia cupa o umoristica, lo stesso motivo appare: l'assenza di comprensione tra le generazioni. Madre e figlia sembrano due modelli antitetici, la cui costruzione si effettua a specchio.

\section{Diventare madre: maternità omosessuali, tra tabù e sovversione delle norme di genere}

Se le protagoniste di Principesse azzurre non assomigliano per niente alle loro madri, la situazione cambia quando diventano madri anche loro. Sin dal primo volume del 2003, l'omogenitorialità femminile è affrontata. Tratta in modo complesso dei conflitti tra norme di sessualità, norme di genere e norme familiari. In Il bambino nel giardino dei girasoli, il desiderio di avere un figlio appare come un sogno, un fantasma di cui si sa che non si può (o non si deve?) realizzare:

«Cosa cerchi?»

«Il nostro bambino. È qui. Dentro di te. Le mie mani sono dove c'è il nostro bambino.»

«Non c'è nessun bambino.»

«Lo desideriamo. Mi basta.»" ${ }^{29}$

Una delle due protagoniste rivela all'altra la sua 'fantasia' adolescenziale: avere un figlio di cui sarebbe il padre («io ero il padre») e prendersi cura di una donna incinta durante la gravidanza, essere presente alle lezioni di preparazione al parto, assistere al parto... Viene presa in giro dalla seconda protagonista: "Ah, non sei rimasta a camminare nervosamente in corridoio fumando una sigaretta dopo l'altra?» La discordanza tra genere e ruolo parentale tradizionale si esprime con la ripresa di uno stereotipo di genere (che corrisponde anche a una realtà storica): l'immagine del

28. Valeria Viganò, Prigioniere, in Principesse azzurre da guardare. Racconti d'amore e di vita di donne tra donne, a cura di Delia Vaccarello, Milano, Mondadori, 2007, p. I73.

29. Iceblues, Il bambino nel giardino dei girasoli, in Principesse azzurre. Racconti d'amore e di vita di donne tra donne, cit., p. 99. 
padre escluso da quella cosa fra donne che sarebbe il parto e che aspetta nel corridoio dandosi un contegno virile. In questo testo però, niente dice che la protagonista vuole essere un uomo, vuole solo essere "padre», come se la volontà di essere genitori senza portare in seno un bambino non potesse essere pensata senza un cambiamento di genere, poiché ci si aspetta da una donna 'vera' che porti il suo bambino. Cambiare genere - nella fantasia - costituisce in realtà un ritorno alla norma molto più di un atto sovversivo.

Questa impossibilità di essere omossessuale e madre non è più così evidente nei volumi successivi, in particolare nel quinto volume in cui il racconto Arcobaleno funge da testo didattico a favore dell'omogenitorialità. Ambientato in un contesto militante (un'associazione di famiglie omogenitoriali), il testo rappresenta famiglie omogenitorali effettive o in divenire. La narratrice-protagonista Daria non è sicura di volere un bambino con la sua compagna Claudia. Durante una breve separazione, Daria organizza a casa un incontro con genitori e bambini e si lascia a poco a poco vincere dal desiderio di fondare la propria famiglia. Con i suoi commenti da 'novellina', la narratrice funge da specchio al lettore/alla lettrice inesperto/a. Mentre si entusiasma del legame tra una bimba e le sue mamme, il discorso ingenuo diventa quasi militante:

Trovo Graziana e Luigia due mamme molto interessanti, sono entrambe spigliate e dalla parola facile. [...] Mentre le guardo provo una strana sensazione: mi sento stupida nell'aver avuto una riserva. [...]

Devo però ammettere, vedere due mamme e una bambina è del tutto naturale. L'entusiasmo della bambina nel gridare «Mammeeeee!», inseguendo una Malù esaltata, rende il piccolo malessere alla bocca dello stomaco sempre più lieve. ${ }^{30}$

Il racconto presenta una visione dell'omogenitorialità molto più positiva di quella del primo volume. La questione di genere dei genitori non si pone più, si può essere "due mamme» senza che l'una diventi un padre. Un happy end conclude d'altronde il racconto: Claudia torna dall'estero di sorpresa subito dopo la partenza degli invitati, abbraccia Daria e si capisce che avranno anche loro un bambino.

Infine l'ultimo racconto dell'ultima raccolta pubblicata si conclude con Ti aspettiamo, testimonianza di una donna incinta al momento della scrittura del testo, da quanto si legge nella biografia finale. Come Arcobaleno, il racconto tratta della difficoltà per una coppia ad andare d'accordo sulla questione della maternità. Nonostante i dubbi, un bimbo 
è concepito, e quella che non voleva un figlio diventa una mamma molto premurosa. Il suo atteggiamento ricorda il 'fantasma' della protagonista del Bambino nel giardino dei girasoli: bada alla sua compagna incinta, assiste alle ecografie... Il nome scelto per la bimba sembra un segno d'intesa con il lettore/la lettrice di Principesse azzurre: si chiamerà Aurora, come la figlia della Bella Addormentata nel Bosco nella versione di Charles Perrault. Le "madri in viaggio», per riprendere il titolo della sezione dedicata alla maternità nel primo volume, sembrano finalmente giunte in porto.

\section{(Auto-)rappresentazioni lesbiche: tra tradizione e ironia}

Nel ventaglio delle figure di donne lesbiche che appaiono nella serie, e malgrado la diversità di personaggi rappresentati (età, livello sociale, origine geografica, aspetto...), è possibile individuare, non una tipologia, ma almeno una ricorrenza di certe figure emblematiche, quali l'Amazzone, la Scrittrice o l'Emarginata. Presenteremo qui soltanto due tipi di rappresentazioni: la coppia butch-fem e la figura dell'androgino.

Il duetto butch-fem è un classico della rappresentazione delle lesbiche in letteratura, tra cui il più celebre è la coppia Stephen-Mary nel Pozzo di solitudine di Marguerite Radclyffe Hall. Lesbica 'mascolina' e lesbica 'femminile': la dicotomia rende problematico il rapporto tra orientamento sessuale e genere, ma anche quello tra norme e trasgressione. Si può pensare l'identità butch in due modi diversi: da un lato, la butch 'imiterebbe' i codici maschili e cercherebbe di ricreare con una fem una parodia di coppia eterosessuale; dall'altro, trasgredendo alla legge del genere femminile di cui il suo corpo dovrebbe essere il supporto, la butch rappresenta un rifiuto delle norme di genere e porta sulla scena pubblica una certa visibilità lesbica. In realtà, la coppia butch-fem mette soprattutto in rilievo il carattere artificiale dell'apparente binarietà del genere (maschio-femmina), come scrive Judith Butler in Gender Trouble ${ }^{3 \mathrm{I}}$.

3I. Judith Butler, Gender Trouble: Feminism and the Subversion of Identity, New York, Routledge, I990, p. I23: "The idea that butch and femme are in some sense 'replicas' or 'copies' of heterosexual exchanges underestimates the erotic significance of these identities as internally dissonant and complex in their resignification of the hegemonic categories by which they are enabled. Lesbian femmes may recall the heterosexual scene, as it were, but also displace it at the same time. In both butch and femme identities, the very notion of an original or natural identity is put into question; indeed, it is precisely that question as it is embodied in these identities that becomes one source of their erotic significance.» 
Nei racconti, coppie butch-fem appaiono in modo regolare, spesso con un umorismo evidente. In uno dei primissimi racconti della collana, Persuasione virtuale, Lara, donna eterosessuale, presenta tutti i simboli della femminilità occidentale: "Lara si sfilò il camice, indossò un'attillata giacchetta in similpelle amaranto sul top metallizzato e i calzoncini neri che "Vogue" prescriveva alle giovani ricercatrici universitarie, $[\ldots]$ e lasciò il laboratorio in un ritmico clicchettio di tacchi alti.»" Interpreta indubbiamente nel racconto la parte della fem. Assidua cliente di un «virtuocentro» in cui si visionano film erotici con una stimolazione fisica prodotta da un ingenioso sistema di elettrodi e di cinghie, Lara vive un'esperienza perturbante quando, dopo una discreta manipolazione di Milena, la hostess del videocentro, il compagno sessuale della cassetta non si rivela essere un uomo come al solito bensì una donna:

La Lara virtuale si gira sul fianco, giocherella con l'erba, si siede; vagamente irrequieta ora, come se aspettasse qualcuno. E in risposta al suo desiderio, dall'intrico dei cespugli esce... una donna, perbacco! Non una poetica ninfa decorativa, non una leggiadra fatina, ma un bel pezzo di ragazza in jeans e camicia a scacchi che senza tanti complimenti si va a sedere accanto a lei. Lara è esterrefatta. ${ }^{33}$

Proprio come la descrizione di Lara aveva tutti gli attributi della fem (tacchi alti, top aderente...), quella della sua amante virtuale ostenta quelli della butch: i jeans e la camicia a scacchi. Si legge il divertimento della voce narrante, che oppone due figure di femminilità mitologica, la ninfa e la fata, alla figura della butch. L'amante virtuale che è servita da intermediaria a Milena per suscitare in Lara un desiderio omosessuale appare come un doppio della hostess. E la descrizione di Milena non manca di ricordare quella della butch del film: "Scorse due scarpe bianche dalla spessa suola di gomma, un paio di jeans sbiaditi che sparivano all'altezza del ginocchio dentro un camice cilestrino, e il dorso della hostess.» ${ }^{34} \mathrm{La}$ dinamica della seduzione sembra usare la dicotomia butch-fem e gli atteggiamenti immaginari che le sono associati quali le coppie azione/passività, cacciatrice/preda. Ma il carattere artificiale del genere notato da Butler è messo chiaramente in evidenza dal racconto quando, prima di recarsi all'appuntamento con Milena, Lara si disfa dei suoi accessori di fem: «Per l'occasione Lara aveva ripudiato con una smorfia le eleganze di "Vogue" e indossato invece i suoi vecchi jeans con una semplice camicetta bianca» ${ }^{35}$;

32. Donatella Maisano, Persuasione virtuale, in Principesse azzurre, cit., p. 39.

33. Ibid., p. 42.

34. Ibid., p. 57.

35. Ibid., p. 60 
allo stesso modo, quando Lara fa finta di resisterle, Milena 'interpreta' la parte del maschio dominante: “"Trovati un'altra scusa, piccola!”, dice con voce roca, alla maniera dei gangster, e interrompe l'assalto.» ${ }^{36}$

Altri racconti si impossessano di queste identità butch-fem, in particolare il racconto Arma segreta nello stesso volume, o la commedia L'estate scorsa giù al ghetto. Rimane sempre centrale l'ottica di una messa in crisi dei luoghi comuni sul genere.

\section{Riscrivere il mito dell'androgino: il terzo sesso omosessuale?}

Un altro modello culturale importante in Principesse azzurre si delinea nel tema classico dell'androgino. Il riferimento a questa figura mitica appare nel pensiero classico, se si pensa al Simposio di Platone, in cui il personaggio di Aristofane propone la seguente spiegazione dell'eros eteroe omossessuale:

Ma innanzitutto bisogna che conosciate la natura della specie umana e quali prove essa ha dovuto attraversare. Nei tempi andati, infatti, la nostra natura non era quella di oggi, ma molto differente. Allora c'erano tra gli uomini tre generi, e non due come adesso, il maschio e la femmina.

Ne esisteva un terzo, che aveva entrambi i caratteri degli altri. Il nome si è conservato sino a noi, ma il genere quello è scomparso. Era l'ermafrodito, un essere che per la forma e il nome aveva caratteristiche sia del maschio che della femmina. Oggi non ci sono più persone di questo genere. ${ }^{37}$

Gli esseri primitivi erano inizialmente forme sferiche doppie (quattro braccia, due sessi, due visi...). Zeus, per punire il loro orgoglio, decise di tagliarli in due e riducendoli a essere maschile o femminile, lasciando ogni nuovo essere unico in cerca della sua metà. Così, gli androgini primitivi, ora separati, provano desideri eterosessuali, poiché la parte femmina ricerca la parte maschio e viceversa, mentre le metà derivate da un essere primitivo maschio ricercano la loro parte maschio (il che spiega il desiderio omosessuale), proprio come «le donne che derivano dall'essere completo di sesso femminile, ebbene queste non si interessano affatto dei maschi: la loro inclinazione le porta piuttosto verso le altre donne ed è da questa specie che derivano le lesbiche» ${ }^{38}$.

Avviene dunque uno spostamento del valore del mito nella raccolta, e più generalmente nel pensiero moderno dell'androginia, in cui l'andro-

36. Ibid., p. 70.

37. Platone, Il simposio, a cura di Mario Trombino, Roma, Armando, 2008, p. 72.

38. Ibid., p. 77. 
gino serve invece a rappresentare l'omosessualità in quanto terzo sesso. L'esempio più interessante appare nel primo racconto del primo volume, Regalo di nozze, in cui una futura sposa assiste con le sue amiche a una presentazione di sex toys tipo riunione-tupperware. La dimostratrice, Carol, è una figura androgina (e lesbica) la cui apparenza riecheggia il mito platonico del taglio degli esseri primitivi:

Si chiamava Carol, veniva dalla California [...]. Le parole le si gonfiavano in gola ed erano difficili da decifrare. Proprio come il suo viso, dolce a destra, severo a sinistra per via della testa completamente rasata a metà e per l'altra metà coperta da una capigliatura bionda, lunga fino alla vita. Un occhio brillava contornato da pesanti linee nere, l'altro mansueto, senza trucco. La bocca invece era divisa, naturalmente pallida. ${ }^{39}$

Carol non è una figura androgina nel senso moderno di individuo il cui genere non è ovvio, ma nel senso classico di essere che è l'uno e l'altro allo stesso tempo, nel senso letterale, anzi geometrico. Altre figure più tradizionali di androgini appaiono nei racconti (la parola «androgina» viene spesso usata nelle descrizioni), come in Lady boy in cui la protagonista narratrice sta di continuo in uno stato intermedio tra maschilità e femminilità, dal punto di vista della società ma anche individuale:

Poi esco dalla doccia e torno davanti allo specchio: un ragazzo abbronzato appare all'improvviso, guardo ancora, sono io, questa è la mia bocca, le mie ciglia, sono io. Rivolgo lo sguardo su di me e sul mio corpo bagnato: le mie tette sono tonde e bianche ma nello specchio sono un ragazzo bello, con la bocca disegnata. ${ }^{40}$

Questa frequente autorappresentazione lesbica tramite la figura dell'androgino necessiterebbe un'analisi profonda. In effetti, la logica sottesa all'analogia tra androginia e omosessualità si spiegherebbe nel fallimento dell'equazione consueta sesso $=$ genere $=$ eterosessualità: se una donna desidera un'altra donna, significa che non è davvero donna e che una sua parte è maschile. L'androgino supera la binarietà maschio/donna e in quanto 'terzo sesso' permette di pensare la trasgressione delle norme abituali di genere, in una specie di naturalizzazione. La ripresa di questa figura potrebbe essere considerata come un modo di perpetuare l'eteronormatività, non ammettendo che una donna potesse desiderarne un'altra senza che il suo genere fosse rimesso in discussione. Il mito permette di non concepire l'orientamento sessuale come indipendente dal genere, e si

39. Maria Rosa Cutrufelli, Regalo di nozze, in Principesse azzurre, cit., p. I9.

40. Federica Tuzi, Lady boy, in Principesse azzurre da guardare. Racconti d'amore e di vita di donne tra donne, cit., p. II2. 
può ritenere la frequente riscrittura del mito nel corpus come una accettazione delle norme di genere. Tuttavia, se si rovescia il problema, l'uso della figura androgina appare a sé stante come un modo di rappresentare il genere come un continuum non binario, certo solo ternario, ma che trascende l'idea di opposizione tra maschile e femminile a favore di una rappresentazione complessa di genere. In questo senso, sarebbe una sovversione della norma. In realtà, lo si vede, è difficilissimo dare un senso univoco e un'analisi definitiva di questo tema, e bisognerebbe analizzare i casi ad uno ad uno, talmente i testi sono diversi. In ogni modo, via via si delinea un'evoluzione dal luogo comune (anche ironico), da una rappresentazione tradizionale piuttosto monolitica (la lesbica fem, la lesbica butch, la lesbica androgina) a una rappresentazione più complessa, che lascia un'importante parte al "pressoché» (come lo indica il titolo dell'ultimo volume, posto sotto il segno del rifiuto delle categorie: «Qui si racconta la vita. Tramontate le ideologie, com'è evidente, la vita sfugge a categorie e rigidità e, insieme a confusioni e stordimenti, ci offre il dono del "pressoché" " ${ }^{41}$ ). La tendenza è manifesta in questa raccolta in cui la questione del genere e del suo carattere multiplo diventa ricorrente: si veda il racconto Il muro di Berlino e noi due, nel quale la narratrice tratta del suo rapporto ambiguo con Nurit, una misteriosa collega venuta dall'Iran o dall'Iraq (non se lo ricorda bene la narratrice), con cui intrattiene un rapporto di intimità ma anche di distanza, una collega la cui ambiguità sessuale (è donna o uomo?) permarrà fino alla fine, come se di fatto il genere contasse poco.

In guisa di conclusione di queste riflessioni sul corpus molto eterogeneo rappresentato dai sette volumi di Principesse azzurre, bisogna soffermarsi brevemente sul valore politico di questi racconti. Un'analisi più lunga andrebbe dedicata ai narratari dei testi: si rivolgono alle lesbiche italiane o a un pubblico più vasto? Si tratta di produrre una letteratura per lesbiche, letteratura pura o letteratura politica il cui scopo sarebbe di dare una visibilità all'omosessualità femminile a un'ampia gamma di lettori/lettrici? Ci limiteremo a sottolineare che, se si leggono le introduzioni successive di Delia Vaccarello, si individua un'evoluzione da una pubblicazione che miri a colmare un vuoto culturale, a fornire alle donne lesbiche modelli letterari positivi di donne lesbiche (che quasi non esistono nella letteratura italiana, abituata a racconti lesbici drammatici ${ }^{42} \mathrm{a}$

4I. Delia Vaccarello, Introduzione: Sortilegio, in Pressoché amanti. Racconti d'amore e di vita di donne tra donne, cit., p. 7.

42. Si può pensare a Benzina di Elena Stancanelli (1998) o al racconto Maria di Dacia Maraini nella raccolta Mio marito (1968). 
una pubblicazione che vorrebbe rendere visibile l'omosessualità femminile a un pubblico anche eterosessuale, cioè combattere l'omofobia che, come le altre forme di discriminazione, nasce dall'ignoranza.

\section{Bibliografia}

Duncan Derek e Ross Charlotte, Reading Allowed: Contemporary Lesbian and Gay Fiction in Italy, in Trends in Contemporary Italian Narrative, 1980-2007, a cura di Ania Gillian e Ann Hallamore Caesar, Cambridge, Cambridge Scholars Publishing, 2007, pp. 90-II3.

Eros up! Principesse azzurre in amore, a cura di Delia Vaccarello, Milano, Mondadori, 2008.

Pinzuti Eleonora, La narrativa lesbica nel contesto europeo. Note al margine, in "Cahiers d'études italiennes. Novecento... e dintorni», 7, 2008, pp. 207-2I7.

Pressoché amanti. Racconti d'amore e di vita di donne tra donne, a cura di Delia Vaccarello, Milano, Mondadori, 2009.

Principesse azzurre. Racconti d'amore e di vita di donne tra donne, a cura di Delia Vaccarello, Milano, Mondadori, 2003.

Principesse azzurre 2. Racconti d'amore e di vita di donne tra donne, a cura di Delia Vaccarello, Milano, Mondadori, 2004.

Principesse azzurre 3. Racconti d'amore e di vita di donne tra donne, a cura di Delia Vaccarello, Milano, Mondadori, 2005.

Principesse azzurre crescono. Racconti d'amore e di vita di donne tra donne, a cura di Delia Vaccarello, Milano, Mondadori, 2006.

Principesse azzurre da guardare. Racconti d'amore e di vita di donne tra donne, a cura di Delia Vaccarello, Milano, Mondadori, 2007. 